\title{
Mitochondrial metabolism in pulmonary hypertension: beyond mountains there are mountains
}

\author{
Miranda K. Culley and Stephen Y. Chan ${ }^{1,2}$ \\ ${ }^{1}$ Center for Pulmonary Vascular Biology and Medicine, Pittsburgh Heart, Lung, Blood, and Vascular Medicine Institute, Division of Cardiology, Department of Medicine, University of Pittsburgh School of \\ Medicine, Pittsburgh, Pennsylvania, USA. ²university of Pittsburgh Medical Center, Pittsburgh, Pennsylvania, USA.
}

\begin{abstract}
Pulmonary hypertension $(\mathrm{PH})$ is a heterogeneous and fatal disease of the lung vasculature, where metabolic and mitochondrial dysfunction may drive pathogenesis. Similar to the Warburg effect in cancer, a shift from mitochondrial oxidation to glycolysis occurs in diseased pulmonary vessels and the right ventricle. However, appreciation of metabolic events in PH beyond the Warburg effect is only just emerging. This Review discusses molecular, translational, and clinical concepts centered on the mitochondria and highlights promising, controversial, and challenging areas of investigation. If we can move beyond the "mountains" of obstacles in this field and elucidate these fundamental tenets of pulmonary vascular metabolism, such work has the potential to usher in much-needed diagnostic and therapeutic approaches for the mitochondrial and metabolic management of PH.
\end{abstract}

\section{Introduction}

Pulmonary hypertension $(\mathrm{PH})$ is an enigmatic disease of the lung vasculature (1). Vessels are marked by endothelial dysfunction, intimal and medial proliferation and hypertrophy, vascular stiffening, in situ thrombosis, and inflammatory infiltration. Panvascular remodeling leads to progressive narrowing and occlusion of the distal arterioles (2), causing increased pulmonary vascular resistance. Mortality in pulmonary arterial hypertension (PAH) is driven by right ventricular (RV) failure, and according to the Registry to Evaluate Early and Long-Term PAH Disease Management (REVEAL Registry), the 5-year survival rates by functional classes I-IV in newly diagnosed patients are $72.2 \%, 71.7 \%, 60.0 \%$, and $43.8 \%$, respectively (3).

$\mathrm{PH}$ results from many disparate triggers and is classified clinically into five groups that determine prognosis and therapy choice (1). A particularly severe subtype, group $1 \mathrm{PAH}$, arises from idiopathic, hereditary causes, toxin exposure, and secondary disease associations, and $\mathrm{PH}$ in groups $2-5$ is associated with myriad comorbidities $(1,4)$. Overlap in the molecular underpinnings of different $\mathrm{PH}$ types remains incompletely defined. Current treatments neither prevent nor reverse the causative pathology. Furthermore, although historically the focus was on pulmonary vessels and the RV, $\mathrm{PH}$ involves extrapulmonary tissues (5-7). Therefore, the development of novel treatments likely hinges on identifying molecular features that unify the multitissue dysfunction that defines $\mathrm{PH}$.

Spanning over two decades, extensive basic, translational, and clinical analyses support a causative link between metabolic

Conflict of interest: SYC has served as a consultant for Actelion, Gilead, Pfizer, and Vivus. Patent applications (SYC) have been filed regarding targeting metabolism in pulmonary hypertension (WO 2017 205595).

Reference information: J Clin Invest. 2018;128(9):3704-3715.

https://doi.org/10.1172/JCI120847. reprogramming and $\mathrm{PH}(8)$. Foundational studies demonstrated a mechanism for increased glycolysis and diminished glucose oxidation in pulmonary vascular cells and RV, paralleling Warburg's observations that proliferating tumor cells display mitochondrial respiratory repression followed by increased glucose uptake and glycolysis $(9,10)$. The master transcription factors HIF-1 and HIF- $2 \alpha$ are critical effectors in this metabolic shift and are stabilized by hypoxic, inflammatory, and metabolic stress - known pathologic triggers of $\mathrm{PH}$ (11-13). Specifically, in $\mathrm{PH}, \mathrm{HIF}-\alpha$-dependent upregulation of pyruvate dehydrogenase kinases 1 and 2 (PDK1/2) inhibits pyruvate dehydrogenase-mediated (PDH-mediated) conversion of pyruvate to acetyl-CoA $(14,15)$. The Warburg effect has been observed in both animal and human models of PH (14, 16-24). Coupled with metabolic dysregulation, mitochondrial dysfunction in $\mathrm{PH}$ results in evasion of mitochondrial-mediated apoptosis (14, $15,25,26)$. Mitochondrial membrane potential is specific for oxidative phosphorylation (OXPHOS) and control of protein flux across the membrane; dissipation of this potential leads to energy collapse and cytochrome $c$ release, driving the intrinsic apoptotic pathway (27). Like in cancer cells, the mitochondria in $\mathrm{PH}$ exhibit hyperpolarized membrane potential and thus resistance to apoptosis $(25,26,28)$. These metabolic perturbations, summarized in Figure 1, have reframed our understanding of $\mathrm{PH}$ pathogenesis (2) and are a basis for the "metabolic theory" of this disease - a concept discussed in recent reviews (29-31). While the Warburg effect has served as a conceptual anchor, the complexities of metabolic reprogramming and mitochondrial dysfunction have become the focus of intensive investigation in PH. This Review will emphasize emerging and controversial molecular concepts beyond the Warburg effect, the potential for and obstacles to clinical translation of current findings, and, finally, how metabolic dysregulation in $\mathrm{PH}$ may inform our clinical framework and improve disease management. 


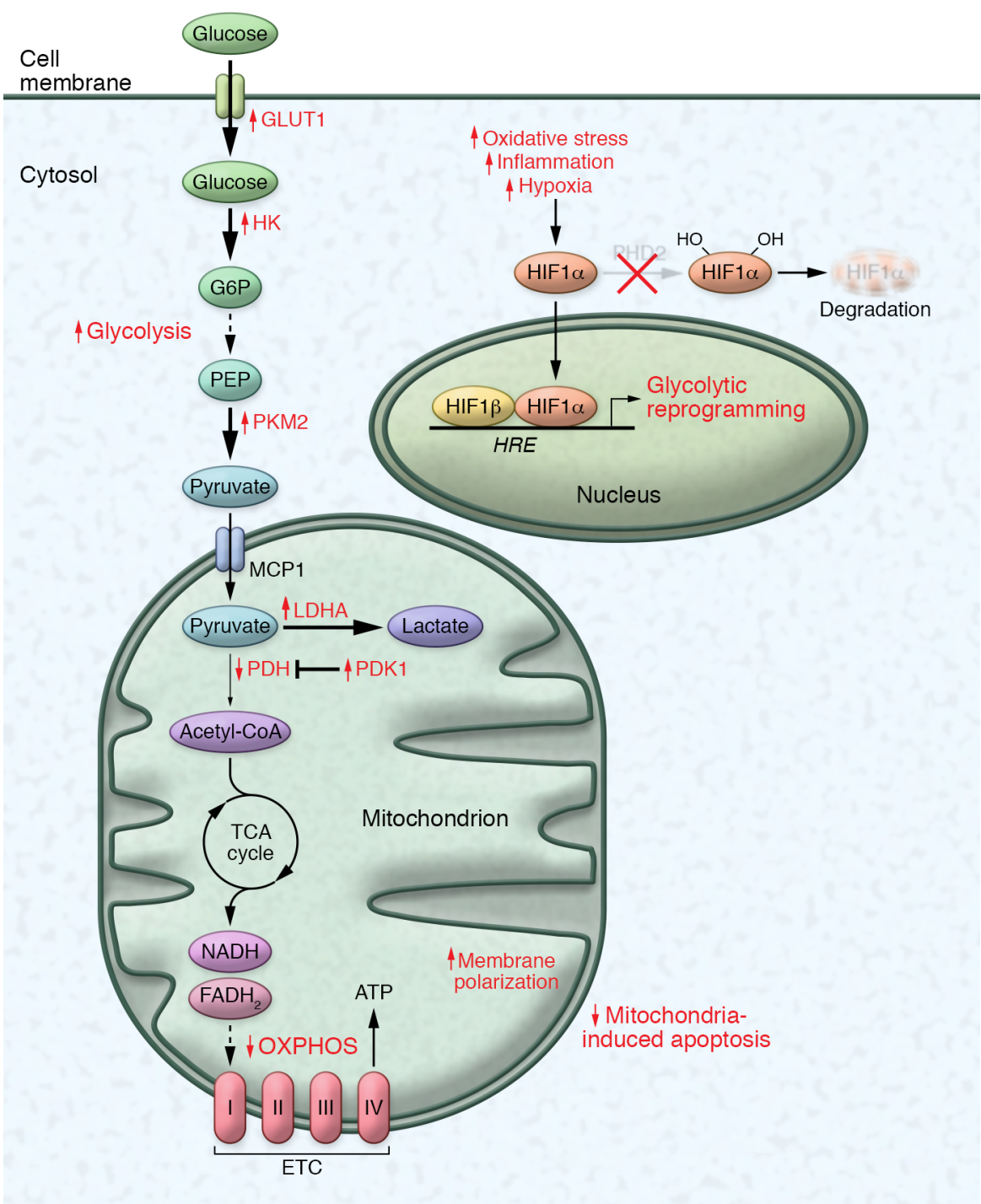

Figure 1. Metabolic pathways disrupted in PH: the Warburg effect. The mitochondria in pulmonary vascular cells and the right ventricle in $\mathrm{PH}$ exhibit decreased oxidative phosphorylation and increased glycolysis, consistent with the Warburg effect. Following a decrease of respiratory activity, transcriptional upregulation and stabilization of HIF- $\alpha$ mediates compensatory glycolytic reprogramming, which includes increased glucose transporter 1 (GLUT1); hexokinase (HK), which traps glucose via conversion to glucose-6-phosphate (G6P); and lactate dehydrogenase (LDHA), which converts pyruvate to lactic acid. At the same time, there is increased activity of pyruvate kinase isoform M2 (PKM2), which slows the production of pyruvate from phosphoenolpyruvate (PEP), and reduced pyruvate dehydrogenase (PDH) activity, driven by elevated pyruvate dehydrogenase kinase (PDK1/2). The Warburg effect promotes cell survival and proliferation, and simultaneous hyperpolarization of the mitochondrial membrane potential promotes evasion of mitochondria-dependent apoptosis. This metabolic switch in pulmonary vascular cells drives extensive remodeling that further results in increased pulmonary vascular resistance and pulmonary artery pressures. ETC, electron transport chain; HRE, hypoxia-response element.

\section{Emerging molecular concepts}

\section{Beyond the Warburg effect}

The molecular drivers of $\mathrm{PH}$ described in Figure 2 build on the Warburg effect, adding complexity to metabolic rewiring and highlighting a role for non-bioenergetic mitochondrial dysfunction in promoting $\mathrm{PH}$. Taking cues from cancer, data demonstrate significant alterations in metabolic programs other than glycolysis and glucose oxidation, including the pentose phosphate pathway (PPP), glutaminolysis, and fatty acid (FA) synthesis and oxidation - all of which may be integral to PH initiation and progression.

Pentose phosphate pathway. While the Warburg effect focuses on glycolysis, the PPP, which yields reductive NADPH and ribose5-phosphate for nucleotide synthesis, is often augmented in parallel with glycolysis. PPP flux is upregulated in pulmonary vascular cells in multiple PH models $(16,32-34)$. Increased activity of the rate-limiting enzyme glucose-6-phosphate dehydrogenase (G6PD) was observed in pulmonary artery smooth muscle cells (PASMCs) of chronically hypoxic rats (32), suggesting that G6PD deficiency may protect against $\mathrm{PH}$ development (35). Increased PPP flux provides defense against oxidative stress and the substrates necessary for rapid growth; therefore, G6PD may represent an enticing target for curbing PASMC hyperproliferation. However, G6PD loss also prevents production of NADPH, a reducing equivalent and critical substrate for NO synthesis (36), suggesting that G6PD inhibition might be detrimental to vasomotor tone in $\mathrm{PH}$. Separately, patients with sickle cell disease, who are at increased risk of developing PH (37), have demonstrated less sickling and hemolysis with increased PPP flux compared with glycolysis. It is not yet known whether targeting aberrant PPP in PH has therapeutic effects, especially in cells heavily reliant on this pathway or exposed to oxidative stress.

Glutaminolysis. Beyond the shift in energy production ascribed to the Warburg effect, insights detail how adequate biomass is made available for vascular cell proliferation in PH. Glutaminolysis is a type of anaplerotic reaction in which tricarboxylic acid (TCA) cycle carbon intermediates are replenished, particularly when rapidly dividing cells require substantial biomass. TCA intermediates from glutaminolysis contribute to amino acid, FA, and de novo purine and pyrimidine biosynthesis (38).

New data support a prominent pathogenic role for glutamine metabolism in PH $(16,39,40)$. Prior work showed that RV cardiomyocyte hypertrophy in monocrotaline-treated rats relies on 


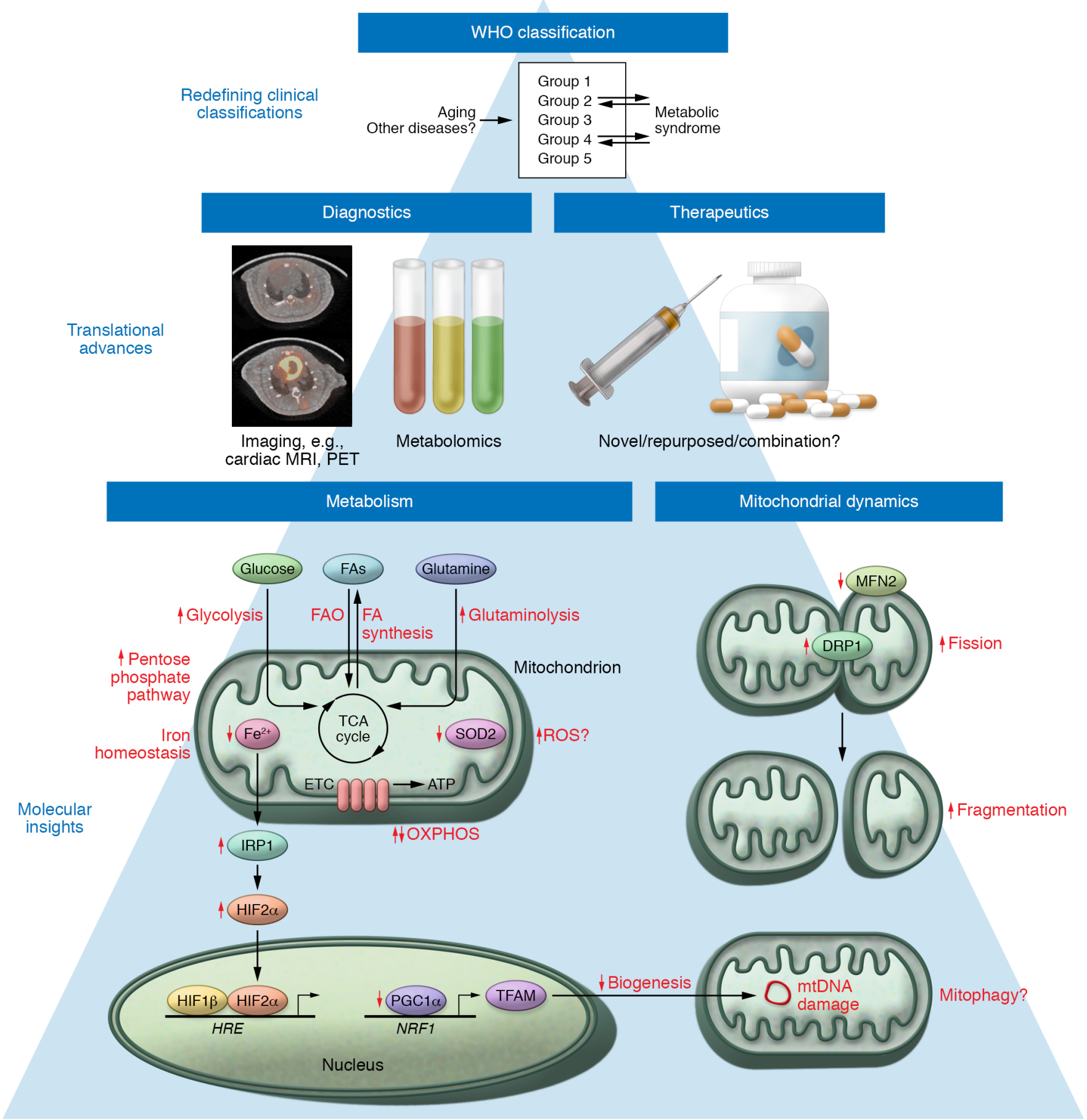

Figure 2. Emerging concepts of mitochondrial dysfunction in PH. Metabolic dysregulation in PH beyond the Warburg effect includes alterations in the pentose phosphate pathway (PPP), glutaminolysis, and FA handling; in certain contexts, it may include an increase of oxidative phosphorylation (a reverse of the Warburg effect); increased reliance on metabolic activities of HIF-2 $\alpha$ and ROS signaling; and profound alterations of iron metabolism. Perturbations in mitochondrial dynamics involve altered mitochondrial biogenesis as well as increased fission and decreased fusion. Dysregulated mitochondrial mass and fragmentation result in metabolic reprogramming and tissue-specific dysfunction typical of PH. A more precise understanding of the complex molecular drivers of PH will inform novel diagnostic technologies and mitochondria-specific therapies. Development of imaging tools such as PET (image courtesy of J. Latoche and C. Anderson, In Vivo Imaging Facility at Hillman Cancer Center, UPMC) and cardiac MRI, high-throughput metabolomic analysis, as well as potential metabolic targeted therapies will be facilitated by a more granular understanding of mitochondrial pathology. Advancements in molecular and translational research may ultimately allow for a redefinition of $\mathrm{PH}$ subtypes through the lens of metabolic dysfunction, with great utility in strategizing appropriate precision medicine therapies. The processes by which other mitochondrial and metabolically driven diseases may be related to $\mathrm{PH}$ are yet to be determined. ETC, electron transport chain; HRE, hypoxia-response element. 
glutaminolysis, which was reversed by the glutamine antagonist 6-diazo-5-oxo-L-norleucine (DON) (39). Furthermore, in pulmonary vascular cells exposed to increasing extracellular matrix stiffness, a recently identified PH trigger (41), mechanoactivation of Yes-associated protein 1 (YAP) and the transcriptional coactivator with a PDZ-binding motif (TAZ, also known as WWTR1), induced the reductive enzyme glutaminase 1 (GLS1), thus promoting glutaminolysis and hyperproliferation (40). Glutaminolytic reprogramming was increased in the vascular lesions of PAH patients and animal models, and multiple vascular cell types in $\mathrm{PH}$ relied on the YAP/TAZ-GLS1 axis (42-45). Pharmacologic inhibition of either YAP or GLS1 also effectively improved rodent PAH. Notably, the YAP/TAZ-GLS1 axis induced glutaminolysis in SIV-induced $\mathrm{PAH}$ primates as well as in HIV-infected PAH patients, suggesting a mechanistic link between glutaminolysis and this enigmatic $\mathrm{PAH}$ subtype. Building on these findings, a separate study demonstrated that elevated glutamate, following GLS1-mediated conversion of glutamine, stimulated NMDA-type glutamate receptors, induced glutamatergic cell-cell signaling in pulmonary vessels, and promoted PH (46). Aberrant glutamine metabolism represents an increasingly promising diagnostic and therapeutic target in $\mathrm{PH}$, given its fundamental roles in promoting proliferative capacity and potentially mediating pathogenic cell-cell interactions.

FA handling. Given that FA oxidation (FAO) accounts for $60 \%-90 \%$ of cellular energy production in ventricular tissue (39), lipid uptake and metabolism in $\mathrm{PH}$ have been primarily studied in the failing RV, but with still incompletely defined results. Patients with PAH display increased circulating free FAs as well as RV-specific accumulation of increased long-chain FAs, triglycerides, and ceramides - hallmarks of lipotoxicity (47-49). Pulmonary artery banding in rats increased RV FAO (50). Conversely, RV FAO was impaired in Bmpr2-mutant mice, whose mutations predispose to $\mathrm{PH}(48,49)$. How FAO is altered in PH is not fully defined. Notably, FAO and glucose oxidation share a reciprocal mechanism, the Randle cycle, in which activation of one inhibits the other (51). Taking advantage of this relationship, pharmacologic inhibition of FAO by trimetazidine or ranolazine increased glucose oxidation and ameliorated RV hypertrophy in animals with PH (50).

Beyond the RV, altered FA metabolism has been observed in diseased pulmonary vasculature. FA synthase, which converts malonyl-CoA to free FA, was upregulated in PASMCs and endothelial cells in PH $(52,53)$. Separately, genetic knockdown of malonyl-CoA decarboxylase, a critical FAO enzyme, or treatment with trimetazidine upregulated glucose oxidation in PASMCs and protected animal models from developing $\mathrm{PH}$ (54). These data suggest that exploiting the Randle cycle could prevent metabolic dysfunction in the pulmonary vasculature, as in the RV. However, efficacy of FAO inhibitors in $\mathrm{PH}$ patients depends on whether FAO-dependent metabolic dysfunction is specific to different pulmonary vascular cell types. Specifically, diminished FAO, due to decreased acetyl-CoA, promoted endothelial-mesenchymal transition (EndoMT) in multiple vascular beds, including the pulmonary vasculature, suggesting that FAO is critical for maintenance of endothelial cell fate and linking it to EndoMT and PH development (55). Separately, endothelial cells in the peripheral circulation rely heavily on FAO for nucleotide synthesis (56); whether this mechanism contributes differentially to pulmonary versus periph- eral endothelium is unclear (57). Additionally, carnitine acyltransferase (CrAT), a "carnitine shuttle" component required for FA transport and metabolism, prevents acyl-CoA accumulation and promotes FAO. Decreased CrAT activity resulted in mitochondrial dysfunction and reduced FA metabolism in the endothelium of lambs with shunt-induced increased pulmonary blood flow, a model of congenital heart disease and PH (58), suggesting that targeting FAO may worsen metabolic dysfunction in this context. Thus, FA metabolic regulation in PH may be cell- and etiologyspecific, potentially complicating direct therapeutic targeting.

\section{A reverse Warburg effect in $\mathrm{PH}$ ?}

While most data support that diminished OXPHOS drives metabolic dysfunction in $\mathrm{PH}$, contemporary evidence reveals that increased glucose oxidation may mediate $\mathrm{PH}$ pathogenesis. Specifically, methamphetamine use, which significantly increases $\mathrm{PH}$ risk and is associated with poor prognosis (59), prevented pulmonary endothelial adaptation to additional environmental stressors like hypoxia, thus increasing OXPHOS, mitochondrial ROS, and DNA damage (60). Separately, mitochondrial activity in platelets from $\mathrm{PAH}$ patients exhibited increased glycolysis and respiratory reserve capacity dependent on FAO (61). This metabolic shift in both glycolysis and respiration correlated with clinical measurements of increased mean pulmonary arterial pressure (MPAP), pulmonary vascular resistance, and RV stroke work index (61). Further study is required to determine whether such metabolic alterations in platelets alter thrombosis formation versus resolution, particularly in chronic thromboembolic PH (group $4 \mathrm{PH}$ ) (62). Together, these data suggest that metabolic reprogramming in PH appears to be context-specific and more complex than what is seen in hypoxic exposure alone. Thus, under certain pathogenic triggers, in specific tissues, or at certain times of disease development, the converse of the classic Warburg effect may be increasingly observed. With the advent of metabolomic and transcriptomic analyses of $\mathrm{PH}$ patients, a systems biology approach may facilitate our understanding of how these metabolic perturbations are interconnected and how future therapies might efficiently reverse these changes.

\section{A central metabolic role for HIF- $2 \alpha$ in PH}

HIF-1 $\alpha$ plays an integral role in promoting and potentiating the Warburg effect; however, its homolog HIF- $2 \alpha$ may be equally or more important in $\mathrm{PH}$, as demonstrated in patients with enhanced HIF$2 \alpha$ signaling. Patients with Chuvash polycythemia, a von HippelLindau-associated disease characterized by HIF- $2 \alpha$ stabilization (63), and patients with HIF-2 $\alpha$ gain-of-function mutations develop PH (64-66). Similarly, there is increased prevalence of a gene variant representing a gain-of-function HIF-2 $\alpha$ mutation in cattle with high-altitude $\mathrm{PH}$, or brisket disease (67). Genetic manipulation in animal models of PH reinforces the importance of HIF- $2 \alpha$. Heterozygous HIF-2 $\alpha$-knockout mice were protected from hypoxic PH (68), and endothelial prolyl hydroxylase 2 (PHD2) knockdown promoted HIF- $2 \alpha$-dependent pulmonary vascular remodeling and $\mathrm{PH}$ in mice $(69,70)$. HIF- $2 \alpha$ exhibits both redundant and unique functions compared with HIF- $1 \alpha$, and HIF- $2 \alpha$ may be more specific to endothelial dysfunction compared with HIF- $1 \alpha$ signaling in PASMCs (71-74). Initial mechanistic studies demonstrated that 
HIF-2 $\alpha$ upregulated arginase, a urea cycle enzyme, thus decreasing arginine availability for NO production and attenuating endothelium-dependent vasodilation $(74,75)$. Furthermore, HIF- $2 \alpha$ modulated mitochondrial superoxide dismutase 2 (SOD2) and thus ROS production in other diseases (76). SOD2 epigenetic silencing has been linked to $\mathrm{PH}$ (77), but whether this controls a robust pathophenotype in $\mathrm{PH}$ is unknown. While additional studies are required to elucidate the unique effects of HIF- $2 \alpha$ versus HIF- $1 \alpha$ or even versus HIF- $3 \alpha$ (78) on mitochondria in $\mathrm{PH}$, current evidence suggests that there may be therapeutic utility in HIF- $2 \alpha$-targeting strategies in the pulmonary vasculature.

\section{Complex alterations in mitochondrial ROS}

Imbalance in multiple types of ROS is causatively linked to $\mathrm{PH}$ development and is reviewed extensively elsewhere (79). The predominant forms of mitochondrial ROS, superoxide, and hydrogen peroxide $\left(\mathrm{H}_{2} \mathrm{O}_{2}\right)$ are of particular interest in $\mathrm{PH}$ because of their role in mitochondrial DNA (mtDNA) damage, cellular signaling, inflammation, and survival (79-81).

The mechanisms of mitochondrial ROS production are not fully defined and are even controversial. In general, a quorum of studies has suggested that the vasculature of nonhypoxic $\mathrm{PAH}$ models exhibits decreased mitochondrial $\mathrm{H}_{2} \mathrm{O}_{2}(14,15)$. Specifically, reduced $\mathrm{H}_{2} \mathrm{O}_{2}$ production due to epigenetic silencing of SOD2 in PASMCs (77) resulted in decreases redox potential, HIF- $1 \alpha$ stabilization, inhibition of redox-sensitive voltage-gated Kv1.5 channels, and increased intracellular calcium, which promoted proliferation (14). In models of persistent $\mathrm{PH}$ of the newborn (PPHN) and chronic hypoxia, decreased endothelial SOD (82) and extracellular SOD (83), respectively, further suggest that decreased $\mathrm{H}_{2} \mathrm{O}_{2}$ production may be a common feature in $\mathrm{PH}$. Conversely, separate studies using a chronic hypoxia model of $\mathrm{PH}$ demonstrated increased mitochondrial superoxide production followed by upregulated HIF-1 $\alpha$ and metabolic reprogramming in PASMCs (84-86). The mechanistic discrepancies in mitochondrial ROS production may depend on experimental conditions, species differences, and perhaps complexities of how pure hypoxic stress may interface with other triggers of PAH. Development of more sensitive, specific, and stable ROS probes and modulatory reagents, particularly in vivo, is needed to understand the temporal (87), subcellular (84), and tissue-, etiology-, and species-specific alterations of mitochondrial ROS in $\mathrm{PH}$.

\section{Bioactive iron deficiency in PH: focus on mitochondria}

Mitochondrial iron metabolism encompasses three major pathways: iron storage, iron-sulfur (Fe-S) cluster formation, and heme synthesis (88). Moreover, an association of iron deficiency with $\mathrm{PH}$ has emerged (89). Because iron is required for PHD2 activity and thus HIF- $\alpha$ degradation, iron deficiency promoted enhanced HIF- $\alpha$ signaling in a rat model of $\mathrm{PH}(90,91)$; similarly, deficiency of ascorbate, another cofactor required for PHD2 activity, was associated with $\mathrm{PH}$ (92). Iron regulatory protein 1 (IRP1) enhances translation of proteins in iron metabolism, including HIF- $2 \alpha$, and has been implicated in PH. Consequently, triggered by low iron, an IRP1-dependent feedback mechanism prevented HIF-2 $\alpha$-driven erythropoiesis, thus worsening iron deficiency as a whole (93). Notably, disruption of the IRP1-HIF-2 $\alpha$ axis and IRP1-dependent feedback promoted PH development (94). Thus, Tempol-induced IRP1 activation has been proposed as an iron-specific therapy for $\mathrm{PH}$ (95). Clinical trials for iron replacement in $\mathrm{PH}$ are also ongoing (96-98). Yet, conversely, data in sickle cell disease have implicated iron overload as a causative trigger in $\mathrm{PH}$ (99). Disease context specificity and/or a need to maintain precise iron homeostasis may underlie these discrepancies, which in turn support caution in excessive iron repletion in $\mathrm{PH}$ patients.

Complementing altered iron homeostasis, deficiency of Fe-S clusters, bioinorganic cofactors required for enzyme redox potential (100), has been found to promote electron transport dysfunction and $\mathrm{PH}$ in experimental rodent models. Specifically, the hypoxiainduced microRNA miR-210 repressed the Fe-S cluster assembly proteins ISCU1 and ISCU2 $(101,102)$, attenuating Fe-S cluster integrity in endothelial cells. Coupled with these mechanistic data, exerciseinduced PH (102) was identified in a patient with rare homozygous mutations of ISCU1/2. Deficiency of other Fe-S cluster assembly proteins may predispose to $\mathrm{PH}$ - an idea reinforced by patients with NFU1 (103) and BOLA3 mutations (104) who exhibit PH. Furthermore, IRP1 is Fe-S cluster-dependent, thus connecting the molecular controls of iron homeostasis in general with Fe-S clusterdriven metabolism and $\mathrm{PH}$.

\section{An imbalance in mitochondrial biogenesis and mitophagy}

Mitochondrial number, or mass, governs cellular bioenergetic capacity and is regulated by biogenesis and mitophagy. Mitochondrial biogenesis is controlled by PPAR $\gamma$ coactivator (PGC1 $\alpha$ ), which activates nuclear respiratory factor 1 (NRF1) and mitochondrial transcription factor A (TFAM) to promote mtDNA genes (105). PH models have demonstrated reduction in mitochondrial biogenesis genes and mass $(22,106,107)$, but mechanistic understanding of biogenesis regulation is lacking. Multiple mediators affect mitochondrial biogenesis in $\mathrm{PH}$, including NO/cGMP signaling (108-110). For example, PPHN modeled in fetal lambs resulted in decreased PGC1 $\alpha$, electron transport chain complex expression, and mtDNA copy number, which was reversed by NO donation (111). Therefore, mitochondrial biogenesis may be restored by treatment with NO derivatives, which are currently being investigated as therapies in PH (112). Separately, biogenesis markers may provide spatiotemporal information about $\mathrm{PH}$ pathogenesis. Monocrotaline-treated rats exhibited reduced expression of PGC1 $\alpha$, NRF1, and TFAM mRNA first in skeletal muscle and later in RV. Moreover, a concomitant decrease in mRNA and protein signatures appeared to differentiate decompensated versus compensated RV failure (113). Temporal perturbations in biogenesis such as these may underlie early-stage skeletal muscle-driven symptoms like exercise intolerance and late-stage heart failure symptoms. Ultimately, detailed temporal- and tissue-specific findings of mitochondrial biogenesis could facilitate stage-specific diagnostic and therapeutic intervention.

Imbalances in mitophagy, the process of removing damaged mitochondria, may also affect mitochondrial mass, but the role of mitophagy and autophagy in $\mathrm{PH}$ is incompletely understood (114). Uncoupling protein 2 (UCP2) is a calcium uniporter that maintains ER-dependent $\mathrm{Ca}^{2+}$ influx to the mitochondria, thus regulating activity of calcium-dependent proteins like $\mathrm{PDH}$, ROS production, and mitochondrial-mediated apoptosis $(28,115,116)$. 
UCP2-deficient endothelial cells exhibited increased mitophagy and reduced mitochondrial mass (117). More work is required to understand how UCP2 and other factors may interconnect processes of biogenesis and mitophagy in $\mathrm{PH}$.

\section{Increased fission and mitochondrial fragmentation}

Mitochondria exist in dynamic networks that are altered by fusion (joining) or fission (division). The dynamin-like GTPases mitofusin 1 and 2 (MFN1/2) mediate fusion, while translocation of activated dynamin-related protein 1 (DRP1) to the outer membrane mediates fission (118). Recent studies demonstrated enhanced mitochondrial fragmentation in PH. Animal models of PH showed increased DRP1 and mitochondrial fragmentation in PASMCs and RV that were reversed by treatment with the DRP1-targeting small molecule mitochondrial division inhibitor (Mdivi-1) or the FAO inhibitor trimetazidine (119-121). DRP1 translocation requires interaction with adaptor proteins (121), including the mitochondrial dynamics proteins MiD49 and MiD51. Elevated MiD49 and MiD51 levels in PASMCs from PAH patients led to enhanced DRP1-mediated fission (122). In parallel, decreased MFN2 expression was observed in diseased PASMCs (123) and skeletal muscle (6). Interestingly, MFN2 reciprocally regulated PGC1 $\alpha$, suggesting a link between diminished mitochondrial mass and increased fragmentation in $\mathrm{PH}$ (123). Mechanisms by which fission and fusion affect other cell types in $\mathrm{PH}$ are unknown, particularly in vivo.

In sum, disparate etiologies of $\mathrm{PH}$ seem to converge at the mitochondria, where certain pathologic features persist, including membrane hyperpolarization, altered ROS and iron handling, increased fragmentation, and decreased mitochondrial mass. Chronic mitochondrial perturbations influence metabolic reprogramming; however, the mechanism of mitochondrial and metabolic dysfunction remains unclear. Beyond genetic mutation and hypoxia, exploration of pathologic drivers in other $\mathrm{PH}$ subtypes may expand our understanding of mitochondrial and subsequent metabolic dysfunction in this disease.

\section{Tissue-specific mitochondrial dysfunction}

Pathologic changes associated with $\mathrm{PH}$ are found in several pulmonary vascular and extrapulmonary cell types. Here, we address nuanced differences in metabolic rewiring in the RV-pulmonary artery (RV-PA) circuit. Additionally, while prior studies focused on tissues with high bioenergetic requirement and mitochondrial content, like PASMCs, we explore the increasingly more relevant roles of mitochondria in other cell types.

The right ventricle. Similar to other cardiopulmonary cell types, RV cardiomyocytes exhibit metabolic rewiring (i.e., increased glycolysis, glutaminolysis, and FAO at the expense of glucose oxidation) in $\mathrm{PH}$, as discussed above $(39,124)$. RV dysfunction may be driven by stepwise, rather than continuous, shifts from normal myocardium to adaptive and maladaptive remodeling. Initial studies have shown distinct metabolic phenotypes between normal, hypertrophic, and decompensated RV failure - namely, increased glycolysis and reduced glucose oxidation as a response to hypoxia during cardiomyocyte hypertrophy and more complex alterations in glucose and FA oxidation in decompensated RV failure (19, 124-126). Additionally, while HIF-1 $\alpha$ expression between the compensated and decompensated RV did not change, HIF-1-dependent genes were differentially expressed (19). In sum, the RV appears to exhibit a distinct stepwise metabolic program, often independent from chronic pressure overload due to pulmonary vascular remodeling (127). Temporal assessment of RV function by novel, noninvasive imaging modalities like PET may be required to better distinguish this metabolic shift and to ensure timing and efficacy of metabolic treatments.

Endothelial cells. Endothelial dysfunction in PH is complex and not fully understood; a prominent model asserts that initial injury and apoptosis precede pathogenic hyperproliferation in $\mathrm{PH}$ (128, 129). Understanding the dynamic phenotype(s) of the pulmonary endothelium has significant clinical implications, potentially facilitating diagnostic determination of disease stage and therapy. Given that endothelial dysfunction in peripheral and pulmonary circuits is defined by rewiring of multiple metabolic pathways $(16,40,58,130,131)$, it is conceivable that mitochondrial changes may provide context for these spatiotemporal alterations. Study of endothelial cells that are genetically predisposed to $\mathrm{PH}$ has provided some clues. Transgenic and heterozygous Bmpr2-mutant mice exhibited increased mtDNA damage in the form of peroxidation-induced DNA adducts (81). In patients with BMPR2 haploinsufficiency, heritable PAH is linked to endothelial metabolic dysfunction (16, 130). BMPR2-mutant endothelial cells displayed increased $\mathrm{p} 53$, mitochondrial biogenesis gene expression, and glycolytic flux, resulting in a proinflammatory state that predisposes to PH. Conversely, BMPR2-deficient endothelial cells exposed to hypoxia followed by reoxygenation demonstrated diminished p53-driven mitochondrial biogenesis coupled with increased mitochondrial fission, mtDNA damage, and apoptosis. Both pathways illustrate how BMPR2 mutation and resultant perturbations in mitochondrial dynamics increase susceptibility to endothelial dysfunction and PH (132). Further interrogation of endothelial mitochondrial function could be instrumental in characterizing the maladaptive shift between early-stage apoptosis and potentially late-stage phenotypes like endothelial-mesenchymal transition $(72,133)$ or hyperproliferation $(2,128,134)$. The study of endothelial cells derived from BMPR2-haploinsufficient inducible pluripotent stem cells may facilitate valuable insights (135-137).

Tissues anatomically distinct from the RV-PA circuit. Cells in circulation - erythrocytes, platelets, and immune cells - are also a focus of study in $\mathrm{PH}$, but how mitochondrial defects in hematopoietic cells influence the pulmonary vasculature is not fully defined. $\mathrm{PH}$ is often a complication of hematologic disorders, particularly anemias (138). In that context, pathologic and age-related drivers of mtDNA mutagenesis are known to prevent mitochondrial elimination from reticulocytes, resulting in deregulated erythroid maturation $(139,140)$. It is unknown whether erythroid progenitor mitochondrial dysfunction could predispose to $\mathrm{PH}$. Similarly, platelets from $\mathrm{PAH}$ patients exhibit increased glycolytic flux and OXPHOS (61), but a causative link between platelet metabolic dysfunction and $\mathrm{PH}$ is not established. Finally, perivascular inflammation is a common feature of $\mathrm{PH}$ (7), and immune cell recruitment to the pulmonary vasculature is under substantial study (141-143). While metabolic reprogramming is linked to macrophage plasticity (144) and $\mathrm{T}$ cell activation (145), a direct connection between that metabolic rewiring and pulmonary vascular inflammation has yet to be defined (146). Furthermore, damage-induced mtDNA release was shown to augment a damage-associated molecular pattern-medi- 
ated (DAMP-mediated) innate immune response in other diseases (147), and DAMP-dependent responses in PH are currently under investigation (81).

The role of progenitor cells in $\mathrm{PH}$ is also an evolving field of study $(148,149)$, and mitochondrial dysfunction in these cells may play a prominent role. Prior data suggested that progenitor cells in PH exhibited altered glucose metabolism; namely, blood outgrowth endothelial cells displayed increased glycolysis (150), and $\mathrm{CD}_{133}{ }^{+}$progenitor cells showed increased G6PD expression and flux through the PPP (33). Shifting endothelial progenitor cells toward mitochondrial OXPHOS was shown to be protective after ischemic injury (151); similar processes may protect against $\mathrm{PH}$.

Given the breadth of metabolic reprogramming across multiple cell types in $\mathrm{PH}$, studies of mitochondrial dysfunction may eventually implicate other anatomically distinct tissues in $\mathrm{PH}$ pathogenesis. In particular, there may be mitochondrial-specific changes in the brain, gut microbiome, and liver, especially in portopulmonary hypertension and iron deficiency (152). Additionally, with the advent of high-throughput methods like single-cell RNA sequencing, nuanced differences in metabolism and mitochondrial function among cells in the same tissue can now be discerned. Lastly, given the multitissue mitochondrial dysfunction in $\mathrm{PH}$, an attractive model may be emerging whereby novel endocrine processes could control anatomic coordination of mitochondrial dysfunction, potentially spearheaded by secretion and uptake of molecular messengers like mitokines (153) or microRNAs, already known to be important in this disease.

\section{Evolving translational technologies}

\section{Diagnostics for metabolic reprogramming}

Imaging. While hemodynamic monitoring remains the gold standard, diagnostic modalities that provide early, accurate, and noninvasive measurement of tissue-specific pathology are needed. The Warburg effect provides the basis for adopting ${ }^{18} \mathrm{~F}$-fluorodeoxyglucose PET (FDG-PET) as a diagnostic tool for PH (154-156). FDG-PET is already used in the clinical setting in other diseases and may provide quantitative spatiotemporal measurement of metabolic reprogramming in RV to complement current hemodynamic testing. Moreover, it may be a future tool for longitudinal measurement of treatment response and targeted drug delivery to metabolically dysregulated tissues. Use of FDG-PET may also prompt adaptation of PET for other metabolically relevant markers in PH-related pathology, such as FAs, amino acids, and TCA metabolites like L-2-hydroxyglutarate, which coordinate pulmonary vascular metabolic responses to reductive stress (157). Metabolic disease progression in the RV may also be followed by four-dimensional flow cardiac MRI, which illustrates RV energy work density and energy dissipation (158), and hyperpolarized cardiac MRI, which can quantify muscle-specific metabolites (159). PET and cardiac MRI can even be coupled in a hybrid technology (160) that may provide an unprecedented view of metabolic perturbations in compensated versus decompensated RV failure, allowing for better diagnostic and prognostic detail in $\mathrm{PH}$ patients.

Plasma metabolomics. Thus far, circulating metabolite screens in $\mathrm{PH}$ have aimed to identify unique profiles in $\mathrm{PH}$ patients and/or distinct PH subtypes for diagnostic and prognostic benefit. Data suggest that certain microRNAs $(102,161)$, plasma acylcarnitines $(47,162)$, glutamate (163), TCA intermediates, amino acids (163, 164), transfer RNA-specific modified nucleosides (163), purines, indoleamines, and indoleamine 2,3-dioxygenase-dependent tryptophan metabolites (165) are differentially expressed in plasma among various cohorts of $\mathrm{PH}$ patients. Moreover, significant metabolite alterations prognosticated risk of death (163) and correlated with hemodynamic measurements (165). High-throughput screening of patient plasma in tandem with other diagnostic tools, like that proposed in the US National Heart, Lung, and Blood Institute's Pulmonary Vascular Disease Phenomics Program (PVDOMICS) initiative (166) and the UK National Cohort Study of PAH (163), could provide group- and stage-specific information and be a step toward implementing precision metabolomic medicine in PH. However, elucidating site-specific metabolic dysregulation via metabolomics may require more invasive transpulmonary blood sampling to ascertain cell source (165). As is the case in recent analyses $(163,166)$, studies also require large-scale cohort validation of existing data. Additional barriers to implementation of routine metabolomic screening include the lack of standardized methods for sampling and detection, controlling among experiments, and interpretation.

Together, next-generation metabolic imaging and metabolomic biomarkers could improve early diagnosis and longitudinal management of $\mathrm{PH}$. Yet a better understanding of the underlying pathology, large-scale analyses correlated with clinical metrics, and removal of high cost and barriers to access are all required for successful implementation.

\section{Prospective mitochondria-targeted therapies}

Mitochondria-specific therapies have the potential to slow or regress $\mathrm{PH}$ development, but several challenges remain. First, tested therapies result in heterogeneous responses in $\mathrm{PH}$ patients. Dichloroacetate (DCA) inhibits PDK2 and upregulates PDH activity, restoring glucose oxidation in PASMCs, endothelial cells, and RV $(50,124)$. Ultimately, this therapy prevented and reversed $\mathrm{PH}$ development in animal models and certain PAH patients $(25,167-169)$. Notably, a subset of patients exhibited variable responses to DCA treatment - heterogeneity that appeared to be dictated by loss-of-function genetic polymorphisms in sirtuin 3 (SIRT3) and UCP2 (168). These data indicate that precision medicine may improve selection of appropriate therapy for patients. Importantly, single-drug metabolic therapy may not be sufficient to reverse $\mathrm{PH}$ pathology given dysregulation of numerous metabolic pathways across multiple affected tissues. For example, ranolazine, the FAO inhibitor, improved PAH symptoms and RV function (57) despite the lack of hemodynamic improvement in MPAP (57). It is possible that ranolazine's efficacy may be limited to the RV, with unclear precedent for interpreting such data and their clinical implications. To address these obstacles more efficiently, we contend that immediate investment is imperative for obtaining -omics-level genetic, transcriptomic, and metabolomic data from clinical trials as combination therapies are tested. Such comprehensive data will be essential in deconvoluting the complexity of these metabolic shifts in $\mathrm{PH}$ patients and will eventually guide creation of more effective mitochondrial-specific therapies, as depicted in Figure 2. 


\section{Redefining metabolic overlap in subtypes of $\mathrm{PH}$}

\section{Metabolic syndrome and $\mathrm{PH}$}

The clinical classification system for $\mathrm{PH}$ defines subtype based on presumed etiology, with consequences for prognosis and therapy. However, given the cumulative data on metabolic and mitochondrial etiologies in $\mathrm{PH}$, we have considered whether this schema must evolve to reflect the intersection or divergence of metabolic phenotypes among PH subtypes. An example of this concept is the association of metabolic syndrome, heart failure with preserved ejection fraction (HFpEF), and $\mathrm{PH}$. Cardiovascular disease has long been linked to metabolic syndrome - dyslipidemia, systemic hypertension, elevated fasting glucose levels, and central obesity (170). Only recently was PH connected to metabolic syndrome $(171,172)$ with patients exhibiting dyslipidemia (173) and insulin resistance $(174,175)$. In addition, SIRT3 (176) and UCP2 (177) polymorphisms are associated with metabolic syndrome and PH $(168,178)$. Tissue-specific SIRT3 knockdown and subsequent metabolic reprogramming were studied in PASMCs (178), endothelial cells (130), fibroblasts (18), and skeletal muscle (179). Interestingly, early restoration of SIRT3 expression in skeletal muscle, the predominant site of insulin-mediated glucose uptake (180), by nitrite or metformin reversed insulin resistance and reduced pulmonary pressures and vascular remodeling in a rodent model of metabolic syndrome and $\mathrm{HFpEF}$ (179). This study confirms the importance of skeletal muscle in metabolically driven $\mathrm{PH}$ and illustrates the role of metabolic syndrome in PH. Yet classification of group $2 \mathrm{PH}$ or $\mathrm{PH}-\mathrm{HFpEF}$ does not reflect this distinct molecular characteristic. Specifically, data support that metabolic syndrome may be present in all $\mathrm{PH}$ subtypes, with a majority of patients in groups 2 and $3 \mathrm{PH}$ (172). Furthermore, HFpEF itself is a diverse clinical entity that encompasses patients with LV diastolic dysfunction without metabolic syndrome. Therefore, we expect that molecular classification of PH subtypes will be an evolving concept, particularly as we learn more about deregulated metabolism in this disease.

\section{Aging and $\mathrm{PH}$}

The age of patients diagnosed with $\mathrm{PH}$ has steadily risen in recent decades (181), and interest in age-associated alterations in pulmonary vascular function has grown $(182,183)$. Moreover, several similarities have emerged between mitochondrial dysfunction during aging and $\mathrm{PH}$ progression. Investigating these perturbations through the lens of aging may deepen and broaden our understanding of the role of mitochondria in PH. For example, stem cell niche and cellular senescence are largely driven by age-dependent alterations in mitochondrial metabolism (184), yet the effect of these processes on the pulmonary vasculature is largely undefined. Without a pathologic aging model of $\mathrm{PH}$ and with a limited number of longitudinal PH databases, the extended experimental timeline has prevented study of aging and $\mathrm{PH}$. These challenges are not insurmountable, and we expect that future exploration of the impact of aging on $\mathrm{PH}$ will also influence its clinical classification and provide valuable opportunities to adapt $\mathrm{PH}$ management in a more elderly population.

\section{Conclusion}

Intensified investigation has revealed the complex metabolic reprogramming and perturbations in mitochondrial dynamics in $\mathrm{PH}$ within and beyond the RV-PA circuit. Yet fundamental questions still remain, including the causal mechanism of mitochondrial dysfunction, how these dysregulated metabolic pathways work synergistically or in opposition to promote disease, and how tissue-specificity and disease stage overlay and define these metabolic programs. Diagnostic and therapeutic technologies based on common mitochondrial features in $\mathrm{PH}$ are promising but require further development in tandem with high-throughput -omics evaluation, given disease heterogeneity. With mitochondrial dysfunction as a central driver of $\mathrm{PH}$, we expect new, causative, and surprising connections to emerge between $\mathrm{PH}$ and other mitochondria-dependent diseases. These links will likely provide a basis for refined classification of $\mathrm{PH}$ subtypes in order to move closer to a precision medicine approach for diagnosis and management of this historically neglected disease.

\section{Acknowledgments}

This work was supported by NIH grants F30 HL139017 (to MKC) and R01 HL124021, HL 122596, HL 138437, and UH2 TR002073 (to SYC), and by American Heart Association grant 18EIA33900027 (to SYC).

Address correspondence to: Stephen Y. Chan, Center for Pulmonary Vascular Biology and Medicine, Pittsburgh Heart, Lung, Blood, and Vascular Medicine Institute, Division of Cardiology, Department of Medicine, University of Pittsburgh Medical Center, 200 Lothrop Street, BST1704.2, Pittsburgh, Pennsylvania 15261, USA. Phone: 412.383.6990; Email: chansy@pitt.edu.
1. Simonneau G, et al. Updated clinical classification of pulmonary hypertension. J Am Coll Cardiol. 2013;62(25 suppl):D34-D41.

2. Stacher E, et al. Modern age pathology of pulmonary arterial hypertension. Am J Respir Crit Care Med. 2012;186(3):261-272.

3. Farber HW, et al. Five-year outcomes of patients enrolled in the REVEAL registry. Chest. 2015;148(4):1043-1054.

4. Lai YC, Potoka KC, Champion HC, Mora AL, Gladwin MT. Pulmonary arterial hypertension: the clinical syndrome. Circ Res. 2014;115(1):115-130.
5. Voelkel NF, et al. Right ventricular function and failure: report of a National Heart, Lung, and Blood Institute working group on cellular and molecular mechanisms of right heart failure. Circulation. 2006;114(17):1883-1891.

6. Batt J, Ahmed SS, Correa J, Bain A, Granton J. Skeletal muscle dysfunction in idiopathic pulmonary arterial hypertension. Am J Respir Cell Mol Biol. 2014;50(1):74-86.

7. Rabinovitch M, Guignabert C, Humbert M, Nicolls MR. Inflammation and immunity in the pathogenesis of pulmonary arterial hypertension. Circ Res. 2014;115(1):165-175.
8. Paulin R, Michelakis ED. The metabolic theory of pulmonary arterial hypertension. Circ Res. 2014;115(1):148-164.

9. Vander Heiden MG, Cantley LC, Thompson CB. Understanding the Warburg effect: the metabolic requirements of cell proliferation. Science. 2009;324(5930):1029-1033.

10. Warburg O. On the origin of cancer cells. Science. 1956;123(3191):309-314.

11. Humbert $M$, et al. Increased interleukin-1 and interleukin- 6 serum concentrations in severe primary pulmonary hypertension. Am J Respir Crit Care Med.1995;151(5):1628-1631. 
12. Ball MK, et al. Regulation of hypoxia-induced pulmonary hypertension by vascular smooth muscle hypoxia-inducible factor-1 $\alpha$. Am J Respir Crit Care Med. 2014;189(3):314-324.

13. Yu AY, et al. Impaired physiological responses to chronic hypoxia in mice partially deficient for hypoxia-inducible factor $1 \alpha$. J Clin Invest. 1999;103(5):691-696.

14. Bonnet S, et al. An abnormal mitochondrialhypoxia inducible factor- $1 \alpha-\mathrm{Kv}$ channel pathway disrupts oxygen sensing and triggers pulmonary arterial hypertension in fawn hooded rats: similarities to human pulmonary arterial hypertension. Circulation. 2006;113(22):2630-2641.

15. Archer SL, Gomberg-Maitland M, Maitland ML, Rich S, Garcia JG, Weir EK. Mitochondrial metabolism, redox signaling, and fusion: a mitochondria-ROS-HIF-1alpha-Kv1.5 O2-sensing pathway at the intersection of pulmonary hypertension and cancer. Am J Physiol Heart Circ Physiol. 2008;294(2):H570-H578.

16. Fessel JP, et al. Metabolomic analysis of bone morphogenetic protein receptor type 2 mutations in human pulmonary endothelium reveals widespread metabolic reprogramming. Pulm Circ. 2012;2(2):201-213.

17. Xu W, et al. Alterations of cellular bioenergetics in pulmonary artery endothelial cells. Proc Natl Acad Sci U S A. 2007;104(4):1342-1347.

18. Zhang $\mathrm{H}$, et al. Metabolic and proliferative state of vascular adventitial fibroblasts in pulmonary hypertension is regulated through a microRNA-124/PTBP1 (Polypyrimidine Tract Binding Protein 1)/pyruvate kinase muscle axis. Circulation. 2017;136(25):2468-2485.

19. Drake JI, et al. Molecular signature of a right heart failure program in chronic severe pulmonary hypertension. Am J Respir Cell Mol Biol. 2011;45(6):1239-1247.

20. Mainguy V, et al. Peripheral muscle dysfunction in idiopathic pulmonary arterial hypertension. Thorax. 2010;65(2):113-117.

21. Li M, et al. Metabolic reprogramming regulates the proliferative and inflammatory phenotype of adventitial fibroblasts in pulmonary hypertension through the transcriptional co-repressor C-terminal binding protein-1. Circulation. 2016;134(15):1105-1121.

22. Malenfant S, et al. Skeletal muscle proteomic signature and metabolic impairment in pulmonary hypertension. JMolMed (Berl). 2015;93(5):573-584.

23. Fijalkowska I, et al. Hypoxia inducible-factor1 $\alpha$ regulates the metabolic shift of pulmonary hypertensive endothelial cells. Am J Pathol. 2010;176(3):1130-1138.

24. Tuder RM, et al. Expression of angiogenesis-related molecules in plexiform lesions in severe pulmonary hypertension: evidence for a process of disordered angiogenesis. J Pathol. 2001;195(3):367-374.

25. McMurtry MS, et al. Dichloroacetate prevents and reverses pulmonary hypertension by inducing pulmonary artery smooth muscle cell apoptosis. Circ Res. 2004;95(8):830-840.

26 . Bonnet S, et al. The nuclear factor of activated $\mathrm{T}$ cells in pulmonary arterial hypertension can be therapeutically targeted. Proc Natl Acad Sci U S A. 2007;104(27):11418-11423.
27. Zamzami N, Kroemer G. The mitochondrion in apoptosis: how Pandora's box opens. Nat Rev Mol Cell Biol. 2001;2(1):67-71.

28. Pak O, et al. Mitochondrial hyperpolarization in pulmonary vascular remodeling. Mitochondrial uncoupling protein deficiency as disease model. Am J Respir Cell Mol Biol. 2013;49(3):358-367.

29. Sutendra G, Michelakis Evangelos D. The metabolic basis of pulmonary arterial hypertension. Cell Metab. 2014;19(4):558-573.

30. Harvey LD, Chan SY. Emerging metabolic therapies in pulmonary arterial hypertension. J Clin Med. 2017;6(4):43.

31. Chan SY, Rubin LJ. Metabolic dysfunction in pulmonary hypertension: from basic science to clinical practice. Eur Respir Rev. 2017;26(146):170094.

32. Chettimada S, Gupte R, Rawat D, Gebb SA, McMurtry IF, Gupte SA. Hypoxia-induced glucose-6-phosphate dehydrogenase overexpression and -activation in pulmonary artery smooth muscle cells: implication in pulmonary hypertension. Am J Physiol Lung Cell Mol Physiol. 2015;308(3):L287-L300.

33. Chettimada S, et al. Glucose-6-phosphate dehydrogenase plays a critical role in hypoxia-induced $\mathrm{CD}_{133^{+}}$progenitor cells self-renewal and stimulates their accumulation in the lungs of pulmonary hypertensive rats. Am J Physiol Lung Cell Mol Physiol. 2014;307(7):L545-L556.

34. Boehme J, et al. Pulmonary artery smooth muscle cell hyperproliferation and metabolic shift triggered by pulmonary overcirculation. Am J Physiol Heart Circ Physiol. 2016;311(4):H944-H957.

35. Dahoui HA, et al. Pulmonary hypertension in children and young adults with sickle cell disease: evidence for familial clustering. Pediatr Blood Canc. 2010;54(3):398-402.

36. Leopold JA, Cap A, Scribner AW, Stanton RC, Loscalzo J. Glucose-6-phosphate dehydrogenase deficiency promotes endothelial oxidant stress and decreases endothelial nitric oxide bioavailability. FASEB J. 2001;15(10):1771-1773.

37. Potoka KP, Gladwin MT. Vasculopathy and pulmonary hypertension in sickle cell disease. Am JPhysiol Lung Cell Mol Physiol. 2015;308(4):L314-LL24.

38. Altman BJ, Stine ZE, Dang CV. From Krebs to clinic: glutamine metabolism to cancer therapy. Nat Rev Cancer. 2016;16(10):619-634

39. Piao L, Fang Y-H, Parikh K, Ryan JJ, Toth PT, Archer SL. Cardiac glutaminolysis: a maladaptive cancer metabolism pathway in the right ventricle in pulmonary hypertension. J Mol Med (Berl). 2013;91(10):1185-1197.

40. Bertero T, et al. Vascular stiffness mechanoactivates YAP/TAZ-dependent glutaminolysis to drive pulmonary hypertension. JClin Invest. 2016;126(9):3313-3335.

41. Liu F, et al. Distal vessel stiffening is an early and pivotal mechanobiological regulator of vascular remodeling and pulmonary hypertension. JCI Insight. 2016;1(8):e86987.

42. Bertero T, et al. Matrix remodeling promotes pulmonary hypertension through feedback mechanoactivation of the YAP/TAZ-miR-130/301 circuit. Cell Rep. 2015;13(5):1016-1032.

43. Bertero T, et al. A YAP/TAZ-miR-130/301 molecular circuit exerts systems-level control of fibrosis in a network of human diseases and physiologic conditions. Sci Rep. 2015;5:18277.

44. Dieffenbach PB, et al. Arterial stiffness induces remodeling phenotypes in pulmonary artery smooth muscle cells via YAP/TAZ-mediated repression of cyclooxygenase-2. Am J Physiol Lung Cell Mol Physiol. 2017;313(3):L628-LL47.

45. Kudryashova TV, et al. HIPPO-integrin-linked kinase cross-talk controls self-sustaining proliferation and survival in pulmonary hypertension. Am J Respir Crit Care Med. 2016;194(7):866-877.

46. Dumas SJ, et al. NMDA-type glutamate receptor activation promotes vascular remodeling and pulmonary arterial hypertension. Circulation. 2018;137(22):2371-2389.

47. Brittain EL, et al. Fatty acid metabolic defects and right ventricular lipotoxicity in human pulmonary arterial hypertension. Circulation. 2016;133(20):1936-1944.

48. Hemnes AR, et al. Evidence for right ventricular lipotoxicity in heritable pulmonary arterial hypertension. Am J Respir Crit Care Med. 2014;189(3):325-334.

49. Talati MH, et al. Mechanisms of lipid accumulation in the bone morphogenetic protein receptor type 2 mutant right ventricle. Am J Respir Crit Care Med. 2016;194(6):719-728.

50. Fang Y-H, et al. Therapeutic inhibition of fatty acid oxidation in right ventricular hypertrophy: exploiting Randle's cycle. J Mol Med (Berl). 2012;90(1):31-43.

51. Randle PJ, Garland PB, Hales CN, Newsholme EA. The glucose fatty-acid cycle. Its role in insulin sensitivity and the metabolic disturbances of diabetes mellitus. Lancet. 1963;1(7285):785-789.

52. Singh N, Manhas A, Kaur G, Jagavelu K, Hanif $\mathrm{K}$. Inhibition of fatty acid synthase is protective in pulmonary hypertension. Br J Pharmacol. 2016;173(12):2030-2045.

53. Singh N, Singh H, Jagavelu K, Wahajuddin M, Hanif K. Fatty acid synthase modulates proliferation, metabolic functions and angiogenesis in hypoxic pulmonary artery endothelial cells. Euro JPharmacol. 2017;815:462-469.

54. Sutendra G, et al. Fatty acid oxidation and malonyl-CoA decarboxylase in the vascular remodeling of pulmonary hypertension. Sci Transl Med. 2010;2(44):44ra58.

55. Xiong J, et al. A metabolic basis for endothelial-to-mesenchymal transition. Mol Cell. 2018;69(4):689-698.e7.

56. Schoors S, et al. Fatty acid carbon is essential for dNTP synthesis in endothelial cells. Nature. 2015;520(7546):192-197.

57. Khan SS, et al. Effects of ranolazine on exercise capacity, right ventricular indices, and hemodynamic characteristics in pulmonary arterial hypertension: a pilot study. Pulm Circ. 2015;5(3):547-556.

58. Sun X, et al. Disruption of endothelial cell mitochondrial bioenergetics in lambs with increased pulmonary blood flow. Antioxid Redox Signal. 2013;18(14):1739-1752.

59. Zamanian RT, et al. Features and outcomes of methamphetamine-associated pulmonary arterial hypertension. Am J Respir Crit Care Med 2018;197(6):788-800.

60. Chen P-I, et al. Amphetamines promote 
mitochondrial dysfunction and DNA damage in pulmonary hypertension. JCI Insight. 2017;2(2):e90427.

61. Nguyen QL, et al. Platelets from pulmonary hypertension patients show increased mitochondrial reserve capacity. JCI Insight. 2017;2(5):e91415.

62. Winter MP, Schernthaner GH, Lang IM. Chronic complications of venous thromboembolism. J Thromb Haemost. 2017;15(8):1531-1540.

63. Hickey MM, et al. The von Hippel-Lindau Chuvash mutation promotes pulmonary hypertension and fibrosis in mice. J Clin Invest. 2010;120(3):827-839.

64. Bushuev V, et al. Endothelin-1, vascular endothelial growth factor and systolic pulmonary artery pressure in patients with Chuvash polycythemia. Haematologica. 2006;91(6):744-749.

65. Formenti F, et al. Cardiopulmonary function in two human disorders of the hypoxia-inducible factor (HIF) pathway: von Hippel-Lindau disease and HIF-2 $\alpha$ gain-of-function mutation. FASEB J. 2011;25(6):2001-2011.

66. Gale DP, Harten SK, Reid CDL, Tuddenham EGD, Maxwell PH. Autosomal dominant erythrocytosis and pulmonary arterial hypertension associated with an activating HIF-2 $\alpha$ mutation. Blood. 2008;112(3):919-921.

67. Newman JH, et al. Increased prevalence of EPAS1 variant in cattle with high-altitude pulmonary hypertension. Nat Commun. 2015;6:6863.

68. Brusselmans K, et al. Heterozygous deficiency of hypoxia-inducible factor-2 $\alpha$ protects mice against pulmonary hypertension and right ventricular dysfunction during prolonged hypoxia. JClin Invest. 2003;111(10):1519-1527.

69. Dai Z, Li M, Wharton J, Zhu MM, Zhao Y-Y. PHD2 deficiency in endothelial cells and hematopoietic cells induces obliterative vascular remodeling and severe pulmonary arterial hypertension in mice and humans through HIF-2 $\alpha$. Circulation. 2016;133(24):2447-2458.

70. Kapitsinou PP, et al. The endothelial prolyl-4-hydroxylase domain 2/hypoxia-inducible factor 2 axis regulates pulmonary artery pressure in mice. Mol Cell Biol. 2016;36(10):1584-1594.

71. Ahmad A, et al. Differential regulation of pulmonary vascular cell growth by hypoxia-inducible transcription factor- $1 \alpha$ and hypoxia-inducible transcription factor-2 $\alpha$. Am J Respir Cell Mol Biol. 2013;49(1):78-85.

72. Tang H, et al. Endothelial HIF-2 $\alpha$ contributes to severe pulmonary hypertension due to endothelial-to-mesenchymal transition. Am J Physiol Lung Cell Mol Physiol. 2018;314(2):L256-LL75.

73. Ema M, Taya S, Yokotani N, Sogawa K, Matsuda Y, Fujii-Kuriyama Y. A novel bHLH-PAS factor with close sequence similarity to hypoxia-inducible factor $1 \alpha$ regulates the VEGF expression and is potentially involved in lung and vascular development. Proc Natl Acad Sci U S A. 1997;94(9):4273-4278.

74. Cowburn AS, et al. HIF2 $\alpha$-arginase axis is essential for the development of pulmonary hypertension. Proc Natl Acad Sci U S A. 2016;113(31):8801-8806.

75. Xu W, et al. Increased arginase II and decreased NO synthesis in endothelial cells of patients with pulmonary arterial hypertension. FASEB J.
2004;18(14):1746-1748.

76. Scortegagna $\mathrm{M}$, et al. Multiple organ pathology, metabolic abnormalities and impaired homeostasis of reactive oxygen species in Epas $1^{-/-}$mice. Nat Genet. 2003;35(4):331.

77. Archer SL, et al. Epigenetic attenuation of mitochondrial superoxide dismutase 2 (SOD2) in pulmonary arterial hypertension: a basis for excessive cell proliferation and a new therapeutic target. Circulation. 2010;121(24):2661-2671.

78. Zhang P, Yao Q, Lu L, Li Y, Chen PJ, Duan C. Hypoxia-inducible factor 3 is an oxygen-dependent transcription activator and regulates a distinct transcriptional response to hypoxia. Cell Rep. 2014;6(6):1110-1121.

79. Tabima DM, Frizzell S, Gladwin MT. Reactive oxygen and nitrogen species in pulmonary hypertension. Free Radic Biol Med. 2012;52(9):1970-1986.

80. Villegas LR, et al. Superoxide dismutase mimetic, MnTE-2-PyP, attenuates chronic hypoxiainduced pulmonary hypertension, pulmonary vascular remodeling, and activation of the NALP3 inflammasome. Antioxid Redox Signal. 2013;18(14):1753-1764.

81. Wauchope OR, et al. Oxidative stress increases M1dG, a major peroxidation-derived DNA adduct, in mitochondrial DNA. Nucleic Acids Res. 2018;46(7):3458-3467.

82. Afolayan AJ, et al. Decreases in manganese superoxide dismutase expression and activity contribute to oxidative stress in persistent pulmonary hypertension of the newborn. Am J Physiol Lung Cell Mol Physiol. 2012;303(10):L870-L879.

83. Nozik-Grayck E, et al. Lung EC-SOD overexpression attenuates hypoxic induction of Egr-1 and chronic hypoxic pulmonary vascular remodeling. Am J Physiol Lung Cell Mol Physiol. 2008;295(3):L422-L430.

84. Waypa GB, et al. Hypoxia triggers subcellular compartmental redox signaling in vascular smooth muscle cells. Circ Res. 2010;106(3):526-535.

85. Waypa GB, et al. Superoxide generated at mitochondrial complex III triggers acute responses to hypoxia in the pulmonary circulation. Am J Respir Crit Care Med. 2013;187(4):424-432.

86. Chi AY, Waypa GB, Mungai PT, Schumacker PT. Prolonged hypoxia increases ROS signaling and RhoA activation in pulmonary artery smooth muscle and endothelial cells. Antioxid Redox Signal. 2010;12(5):603-610.

87. Pak O, et al. Impact of the mitochondria-targeted antioxidant MitoQ on hypoxia-induced pulmonary hypertension [published online ahead of print February 1, 2018]. Eur Respir J. https://doi.org/10.1183/13993003.01024-2017.

88. Richardson DR, et al. Mitochondrial iron trafficking and the integration of iron metabolism between the mitochondrion and cytosol. Proc Natl Acad Sci U S A. 2010;107(24):10775-10782.

89. Rhodes CJ, et al. Iron deficiency and raised hepcidin in idiopathic pulmonary arterial hypertension: clinical prevalence, outcomes, and mechanistic insights. J Am Coll Cardiol. 2011;58(3):300-309.

90. Cotroneo E, et al. Iron homeostasis and pulmonary hypertension: iron deficiency leads to pulmonary vascular remodeling in the rat. Circ Res. 2015;116(10):1680-1690
91. Schofield CJ, Ratcliffe PJ. Oxygen sensing by HIF hydroxylases. Nat Rev Mol Cell Biol. 2004;5(5):343-354.

92. Kupari M, Rapola J. Reversible pulmonary hypertension associated with vitamin C deficiency. Chest. 2012;142(1):225-227.

93. Sanchez M, Galy B, Muckenthaler MU, Hentze MW. Iron-regulatory proteins limit hypoxia-inducible factor- $2 \alpha$ expression in iron deficiency. Nat Struct Mol Biol. 2007;14(5):420-426.

94. Ghosh MC, et al. Deletion of iron regulatory protein 1 causes polycythemia and pulmonary hypertension in mice through translational derepression of HIF2 $\alpha$. Cell Metab. 2013;17(2):271-281.

95. Ghosh MC, et al. Tempol-mediated activation of latent iron regulatory protein activity prevents symptoms of neurodegenerative disease in IRP2 knockout mice. Proc Natl Acad Sci U S A. 2008;105(33):12028-12033.

96. Rhodes CJ, Wharton J, Howard L, Gibbs JS, Vonk-Noordegraaf A, Wilkins MR. Iron deficiency in pulmonary arterial hypertension: a potential therapeutic target. Eur Respir J. 2011;38(6):1453-1460.

97. Ruiter $\mathrm{G}$, et al. Intravenous iron therapy in patients with idiopathic pulmonary arterial hypertension and iron deficiency. Pulm Circ. 2015;5(3):466-472.

98. Frise MC, et al. Clinical iron deficiency disturbs normal human responses to hypoxia. JClin Invest. 2016;126(6):2139-2150.

99. Wang $X$, et al. Heme-bound iron activates placenta growth factor in erythroid cells via erythroid Krüppel-like factor. Blood. 2014;124(6):946-954.

100.Lill R. Function and biogenesis of iron-sulphur proteins. Nature. 2009;460(7257):831-838.

101.Chan SY, Zhang YY, Hemann C, Mahoney CE, Zweier JL, Loscalzo J. MicroRNA-210 controls mitochondrial metabolism during hypoxia by repressing the iron-sulfur cluster assembly proteins ISCU1/2. Cell Metab. 2009;10(4):273-284.

102. White $\mathrm{K}$, et al. Genetic and hypoxic alterations of the microRNA-210-ISCU1/2 axis promote iron-sulfur deficiency and pulmonary hypertension. EMBO Mol Med. 2015;7(6):695-713.

103. Navarro-Sastre A, et al. A fatal mitochondrial disease is associated with defective NFU1 function in the maturation of a subset of mitochondrial Fe-S proteins. Am JHum Genet. 2011;89(5):656-667.

104.Ahting U, et al. Clinical, biochemical, and genetic spectrum of seven patients with NFU1 deficiency. Front Genet. 2015;6:123.

105. Vega RB, Horton JL, Kelly DP. Maintaining ancient organelles: mitochondrial biogenesis and maturation. Circ Res. 2015;116(11):1820-1834.

106.Ye JX, Wang SS, Ge M, Wang DJ. Suppression of endothelial PGC-1 $\alpha$ is associated with hypoxia-induced endothelial dysfunction and provides a new therapeutic target in pulmonary arterial hypertension. Am J Physiol Lung Cell Mol Physiol. 2016;310(11):L1233-L1242.

107. Gomez-Arroyo J, et al. Metabolic gene remodeling and mitochondrial dysfunction in failing right ventricular hypertrophy secondary to pulmonary arterial hypertension. Circ Heart Fail. 2013;6(1):136-144.

108. Yeligar SM, et al. PPAR $\gamma$ regulates mitochondrial structure and function and human pulmonary 
artery smooth muscle cell proliferation. $A m \mathrm{~J}$ Respir Cell Mol Biol. 2018;58(5):648-657.

109.Zurlo G, et al. Sirtuin 1 regulates pulmonary artery smooth muscle cell proliferation: role in pulmonary arterial hypertension. J Hypertens. 2018;36(5):1164-1177.

110. Nisoli E, et al. Mitochondrial biogenesis by NO yields functionally active mitochondria in mammals. Proc Natl Acad Sci U S A. 2004;101(47):16507-16512.

111. Afolayan AJ, et al. Decreased endothelial nitric oxide synthase expression and function contribute to impaired mitochondrial biogenesis and oxidative stress in fetal lambs with persistent pulmonary hypertension. Am J Physiol Lung Cell Mol Physiol. 2016;310(1):L40-L49.

112. Simon MA, et al. Acute hemodynamic effects of inhaled sodium nitrite in pulmonary hypertension associated with heart failure with preserved ejection fraction. JCI Insight. 2016;1(18):e89620.

113. Enache I, et al. Skeletal muscle mitochondrial dysfunction precedes right ventricular impairment in experimental pulmonary hypertension. Mol Cell Biochem. 2013;373(1-2):161-170.

114. Cloonan SM, Choi AMK. Mitochondria in lung disease. J Clin Invest. 2016;126(3):809-820.

115. Dromparis P, Paulin R, Sutendra G, Qi AC, Bonnet S, Michelakis ED. Uncoupling protein 2 deficiency mimics the effects of hypoxia and endoplasmic reticulum stress on mitochondria and triggers pseudohypoxic pulmonary vascular remodeling and pulmonary hypertension. Circ Res. 2013;113(2):126-136.

116. Sutendra G, et al. The role of Nogo and the mitochondria-endoplasmic reticulum unit in pulmonary hypertension. Sci Transl Med. 2011;3(88):88ra55

117. Haslip M, et al. Endothelial uncoupling protein 2 regulates mitophagy and pulmonary hypertension during intermittent hypoxia. Arterioscler Thromb Vasc Biol. 2015;35(5):1166-1178.

118. Youle RJ, van der Bliek AM. Mitochondrial fission, fusion, and stress. Science. 2012;337(6098):1062-1065.

119. Parra V, et al. Inhibition of mitochondrial fission prevents hypoxia-induced metabolic shift and cellular proliferation of pulmonary arterial smooth muscle cells. Biochim Biophys Acta. 2017;1863(11):2891-2903.

120. Marsboom G, et al. Dynamin-related protein 1-mediated mitochondrial mitotic fission permits hyperproliferation of vascular smooth muscle cells and offers a novel therapeutic target in pulmonary hypertension. Circ Res. 2012;110(11):1484-1497.

121. Tian L, et al. Ischemia-induced Drp1 and Fis1-mediated mitochondrial fission and right ventricular dysfunction in pulmonary hypertension. J Mol Med. 2017;95(4):381-393.

122. Chen $\mathrm{KH}$, et al. Epigenetic dysregulation of the Drp1 binding partners MiD49 and MiD51 increases mitotic mitochondrial fission and promotes pulmonary arterial hypertension: mechanistic and therapeutic implications [published online ahead of print February 5, 2018]. Circulation. https://doi. org/10.1161/CIRCULATIONAHA.117.031258.

123. Ryan JJ, et al. PGC1 $\alpha$-mediated mitofusin-2 deficiency in female rats and humans with pulmo- nary arterial hypertension. Am J Respir Crit Care Med. 2013;187(8):865-878.

124. Piao L, et al. The inhibition of pyruvate dehydrogenase kinase improves impaired cardiac function and electrical remodeling in two models of right ventricular hypertrophy: resuscitating the hibernating right ventricle. J Mol Med. 2010;88(1):47-60.

125. Paulin R, et al. A miR-208-Mef2 axis drives the decompensation of right ventricular function in pulmonary hypertension. Circ Res. 2015;116(1):56-69.

126.Sutendra G, et al. A metabolic remodeling in right ventricular hypertrophy is associated with decreased angiogenesis and a transition from a compensated to a decompensated state in pulmonary hypertension. J Mol Med. 2013;91(11):1315-1327.

127. Bogaard HJ, et al. Chronic pulmonary artery pressure elevation is insufficient to explain right heart failure. Circulation. 2009;120(20):1951-1960.

128.Sakao S, Taraseviciene-Stewart L, Lee JD, Wood K, Cool CD, Voelkel NF. Initial apoptosis is followed by increased proliferation of apoptosis-resistant endothelial cells. FASEB J. 2005;19(9):1178-1180.

129.Xu W, Erzurum SC. Endothelial cell energy metabolism, proliferation, and apoptosis in pulmonary hypertension. Compr Physiol. 2011;1(1):357-372.

130. Egnatchik RA, et al. Dysfunctional BMPR2 signaling drives an abnormal endothelial requirement for glutamine in pulmonary arterial hypertension. Pulm Circ. 2017;7(1):186-199.

131. Rohlenova K, Veys K, Miranda-Santos I, De Bock K, Carmeliet P. Endothelial cell metabolism in health and disease. Trends Cell Biol. 2018;28(3):224-236.

132. Diebold I, et al. BMPR2 preserves mitochondrial function and DNA during reoxygenation to promote endothelial cell survival and reverse pulmonary hypertension. Cell Metab. 2015;21(4):596-608.

133. Suzuki $\mathrm{T}$, et al. Isolation and characterization of endothelial-to-mesenchymal transition cells in pulmonary arterial hypertension. Am J Physiol Lung Cell Mol Physiol. 2018;314(1):L118-L126.

134. Masri FA, et al. Hyperproliferative apoptosis-resistant endothelial cells in idiopathic pulmonary arterial hypertension. Am J Physiol Lung Cell Mol Physiol. 2007;293(3):L548-L554.

135. Gu M, et al. Patient-specific iPSC-derived endothelial cells uncover pathways that protect against pulmonary hypertension in BMPR2 mutation carriers. Cell Stem Cell. 2017;20(4):490-504.e5.

136. Rhodes CJ, et al. RNA sequencing analysis detection of a novel pathway of endothelial dysfunction in pulmonary arterial hypertension. Am J Respir Crit Care Med. 2015;192(3):356-366.

137. Kiskin FN, et al. Contributions of BMPR2 mutations extrinsic factors to cellular phenotypes of pulmonary arterial hypertension revealed by iPSC modeling [published online ahead of print March 16, 2018]. Am J Respir Crit Care Med. https://doi.org/10.1164/rccm.201801-0049LE.

138. Machado RF, Farber HW. Pulmonary hypertension associated with chronic hemolytic anemia and other blood disorders. Clin Chest Med.
2013;34(4):739-752.

139. Ahlqvist KJ, et al. MtDNA mutagenesis impairs elimination of mitochondria during erythroid maturation leading to enhanced erythrocyte destruction. Nat Commun. 2015;6:6494.

140.Li-Harms X, et al. Mito-protective autophagy is impaired in erythroid cells of aged mtDNA-mutator mice. Blood. 2015;125(1):162-174.

141. Frid MG, et al. Hypoxia-induced pulmonary vascular remodeling requires recruitment of circulating mesenchymal precursors of a monocyte/macrophage lineage. Am J Pathol. 2006;168(2):659-669.

142.Savai R, et al. Immune and inflammatory cell involvement in the pathology of idiopathic pulmonary arterial hypertension. Am J Respir Crit Care Med. 2012;186(9):897-908.

143. El Kasmi KC, et al. Adventitial fibroblasts induce a distinct proinflammatory/profibrotic macrophage phenotype in pulmonary hypertension. JImmunol. 2014;193(2):597-609.

144. El Kasmi KC, Stenmark KR. Contribution of metabolic reprogramming to macrophage plasticity and function. Semin Immunol. 2015;27(4):267-275.

145. Wang R, et al. The transcription factor Myc controls metabolic reprogramming upon T lymphocyte activation. Immunity. 2011;35(6):871-882.

146.D'Alessandro A, et al. Hallmarks of pulmonary hypertension: mesenchymal and inflammatory cell metabolic reprogramming. Antioxid Redox Signal. 2018;28(3):230-250.

147. Oka T, et al. Mitochondrial DNA that escapes from autophagy causes inflammation and heart failure. Nature. 2012;485(7397):251-255.

148. Sheikh AQ, Misra A, Rosas IO, Adams RH, Greif DM. Smooth muscle cell progenitors are primed to muscularize in pulmonary hypertension. Sci Transl Med. 2015;7(308):308ra159.

149. Yan L, et al. Bone marrow-derived cells contribute to the pathogenesis of pulmonary arterial hypertension. Am J Respir Crit Care Med. 2016;193(8):898-909.

150. Caruso $P$, et al. Identification of microRNA-124 as a major regulator of enhanced endothelial cell glycolysis in pulmonary arterial hypertension via PTBP1 (polypyrimidine tract binding protein) and pyruvate kinase M2. Circulation. 2017;136(25):2451-2467.

151. Xu S, et al. E2F1 suppresses oxidative metabolism and endothelial differentiation of bone marrow progenitor cells. Circ Res. 2018;122(5):701-711.

152. Koch CD, Gladwin MT, Freeman BA, Lundberg JO, Weitzberg E, Morris A. Enterosalivary nitrate metabolism and the microbiome: intersection of microbial metabolism, nitric oxide and diet in cardiac and pulmonary vascular health. Free Radic Biol Med. 2017;105:48-67.

153. Kim KH, et al. Autophagy deficiency leads to protection from obesity and insulin resistance by inducing Fgf21 as a mitokine. Nat Med. 2013;19(1):83-92.

154. Lundgrin EL, et al. Fasting 2-deoxy-2-[18F]fluoro-D-glucose positron emission tomography to detect metabolic changes in pulmonary arterial hypertension hearts over 1 year. Ann Am Thorac Soc. 2013;10(1):1-9.

155. Marsboom G, et al. Lung ${ }^{18} \mathrm{~F}$-fluorodeoxyglucose positron emission tomography for diagnosis and 
monitoring of pulmonary arterial hypertension. Am J Respir Crit Care Med. 2012;185(6):670-679.

156.Zhao L, et al. Heterogeneity in lung (18)FDG uptake in pulmonary arterial hypertension: potential of dynamic (18)FDG positron emission tomography with kinetic analysis as a bridging biomarker for pulmonary vascular remodeling targeted treatments. Circulation. 2013;128(11):1214-1224.

157. Oldham WM, Clish CB, Yang Y, Loscalzo J. Hypoxia-mediated increases in L-2-hydroxyglutarate coordinate the metabolic response to reductive stress. Cell Metab. 2015;22(2):291-303.

158. Barker AJ, et al. Four-dimensional flow assessment of pulmonary artery flow and wall shear stress in adult pulmonary arterial hypertension: results from two institutions. Magn Reson Med. 2015;73(5):1904-1913.

159. Schroeder MA, et al. Hyperpolarized (13)C magnetic resonance reveals early- and late-onset changes to in vivo pyruvate metabolism in the failing heart. Eur J Heart Fail. 2013;15(2):130-140.

160. Quail MA, Sinusas AJ. PET-CMR in heart failure - synergistic or redundant imaging? Heart Fail Rev. 2017;22(4):477-489.

161. Bertero T, et al. Systems-level regulation of microRNA networks by miR-130/301 promotes pulmonary hypertension. J Clin Invest. 2014;124(8):3514-3528.

162.Luo N, et al. Plasma acylcarnitines are associated with pulmonary hypertension. Pulm Circ. 2017;7(1):211-218.

163. Rhodes CJ, et al. Plasma metabolomics implicates modified transfer RNAs and altered bioenergetics in the outcomes of pulmonary arterial hypertension. Circulation. 2017;135(5):460-475.

164. Bujak R, et al. New biochemical insights into the mechanisms of pulmonary arterial hypertension in humans. PLoS One. 2016;11(8):e0160505.
165. Lewis GD, et al. Metabolic profiling of right ventricular-pulmonary vascular function reveals circulating biomarkers of pulmonary hypertension. JAm Coll Cardiol. 2016;67(2):174-189.

166. Hemnes AR, et al. PVDOMICS: a multi-center study to improve understanding of pulmonary vascular disease through phenomics. Circ Res. 2017;121(10):1136-1139.

167. Bonnet $\mathrm{S}$, et al. A mitochondria- $\mathrm{K}^{+}$channel axis is suppressed in cancer and its normalization promotes apoptosis and inhibits cancer growth. Cancer Cell. 2007;11(1):37-51.

168. Michelakis ED, et al. Inhibition of pyruvate dehydrogenase kinase improves pulmonary arterial hypertension in genetically susceptible patients. Sci Transl Med. 2017;9(413):eaao4583.

169. Michelakis ED, et al. Dichloroacetate, a metabolic modulator, prevents and reverses chronic hypoxic pulmonary hypertension in rats: role of increased expression and activity of voltage-gated potassium channels. Circulation. 2002;105(2):244-250.

170. Mottillo S, et al. The metabolic syndrome and cardiovascular risk a systematic review and meta-analysis. J Am Coll Cardiol. 2010;56(14):1113-1132.

171. Gopal DM, et al. Impaired right ventricular hemodynamics indicate preclinical pulmonary hypertension in patients with metabolic syndrome. JAm Heart Assoc. 2015;4(3):e001597.

172. Ussavarungsi K, Thomas CS, Burger CD. Prevalence of metabolic syndrome in patients with pulmonary hypertension. Clin Respir J. 2017;11(6):721-726.

173. Heresi GA, Aytekin M, Newman J, DiDonato J, Dweik RA. Plasma levels of high-density lipoprotein cholesterol and outcomes in pulmonary arterial hypertension. Am J Respir Crit Care Med. 2010;182(5):661-668.

174. Zamanian RT, et al. Insulin resistance in pul- monary arterial hypertension. Eur Respir J. 2009;33(2):318-324.

175. Pugh ME, Robbins IM, Rice TW, West J, Newman $\mathrm{JH}$, Hemnes AR. Unrecognized glucose intolerance is common in pulmonary arterial hypertension. J Heart Lung Transplant. 2011;30(8):904-911.

176. Hirschey MD, et al. SIRT3 deficiency and mitochondrial protein hyperacetylation accelerate the development of the metabolic syndrome. Mol Cell. 2011;44(2):177-190.

177. Jia JJ, Zhang X, Ge CR, Jois M. The polymorphisms of UCP2 and UCP3 genes associated with fat metabolism, obesity and diabetes. Obes Rev. 2009;10(5):519-526.

178. Paulin R, et al. Sirtuin 3 deficiency is associated with inhibited mitochondrial function and pulmonary arterial hypertension in rodents and humans. Cell Metab. 2014;20(5):827-839.

179. Meng Q, et al. Development of a mouse model of metabolic syndrome, pulmonary hypertension, and heart failure with preserved ejection fraction. Am J Respir Cell Mol Biol. 2017;56(4):497-505.

180.DeFronzo RA, Tripathy D. Skeletal muscle insulin resistance is the primary defect in type 2 diabetes. Diabetes Care. 2009;32(suppl 2):S157-S163.

181. Hyduk A, Croft JB, Ayala C, Zheng K, Zheng ZJ, Mensah GA. Pulmonary hypertension surveillance - United States, 1980-2002. MMWR Surveill Summ. 2005;54(5):1-28.

182. Lam CS, Borlaug BA, Kane GC, Enders FT, Rodeheffer RJ, Redfield MM. Age-associated increases in pulmonary artery systolic pressure in the general population. Circulation. 2009;119(20):2663-2670.

183. Kane GC, et al. Impact of age on pulmonary artery systolic pressures at rest and with exercise. Echo Res Pract. 2016;3(2):53-61.

184.Sun N, Youle RJ, Finkel T. The mitochondrial basis of aging. Mol Cell. 2016;61(5):654-666. 